\title{
The Performance of Estimators for Generalization of Crack Distribution
}

\author{
SUPITCHA MAMUANGBON, KAMON BUDSABA \\ Department of Mathematics and Statistics, Thammasat University, Pathum Thani, THAILAND \\ ANDREI VOLODIN \\ Department of Mathematics and Statistics, University of Regina, Saskatchewan, CANADA
}

\begin{abstract}
In this research, we propose a new four parameter family of distributions called Generalized Crack distribution. We generalizes the family three parameter Crack distribution. The Generalized Crack distribution is a mixture of two parameter Inverse Gaussian distribution, Length-Biased Inverse Gaussian distribution, Twice Length-Biased Inverse Gaussian distribution, and adding one more weight parameter $q$. It is a special case for $p+q+r=1$, where $\lambda>0, \theta>0,0 \leq p \leq 1,0 \leq q \leq 1$ and $p+q+r=1$ is the weighted parameter. We investigate the properties of Generalized Crack distribution including first four moments, parameters estimation by using the maximum likelihood estimators and method of moment estimation. Evaluate the performance of the estimators by using bias. The results of simulation are presented in numerically and graphically.
\end{abstract}

Keywords - Crack distribution, Inverse Gaussian distribution, Length Biased Inverse Gaussian Distribution, Birnbaum-Saunders distribution.

Received: December 14, 2020. Revised: March 10, 2021. Accepted: March 26, 2021. Published: April 12, 2021.

\section{Introduction}

The lifetime distribution provides an advantages and applied information to users or practitioners to protect damages of medical, finance, manufacturing, systems, industry, and machines that occur after the lifetime is terminated. The serious injury or death may be happened if the user not know the lifetime of their items, machines or systems.

The well known lifetime distributions in survival analysis are Birnbaum-Saunders (BS), Inverse Gaussian (IG), Length Biased Inverse Gaussian (LB) and Crack distributions (CR), Exponential, Log-Normal, Extreme Value, Weibull.

The distribution is widely used to be the lifetime distributions in many filed. The distributions had been studied for long time. For Inverse Gaussian distribution [1-3], Shuster (1968) [4] used the tables of the Standard Normal distribution and logarithms to get the exact probabilities for an Inverse Gaussian distribution. Chhikara and Folks (1989) [5] provided the relationship among Inverse Gaussian distribution and $\chi^{2}$ and $F$ distributions toapplied with the Sampling Theory. They also mentioned that the Inverse Gaussian distribution describes the distribution of the time it takes a Brownian motion while the normal distribution express the distance traveled at time fixed by the standard Brownian motion. Chaubey et al. (2014) [6] proved that under the scale transformation, the likelihood ratio test for one sided hypotheses in the Inverse Gaussian family is the uniformly most powerful invariant test.

The length biased in version of the Inverse Gaussian distribution was studied in [7] and [8]. Patil and Rao (1977) [9] proposed the special weighted distribution of length biased in version of the Inverse Gaussian distribution.

The Crack distribution was introduced by Jorgensen et al. (1991) [10]. Gupta and Akman (1995) [11] studied the Bayesian estimation of Crack distribution. Gupta and Akman (1995) [12] discussed that the Crack distribution is also known as the Inverse Gaussian Mixture distribution. Volodin and
Dzhungurova (2000) [13] proposed the five-parameter family of called General Crack distributions, which including to the Inverse Gaussian Mixture distribution, normal distribution, the Inverse Gaussian distribution, and the Birnbaum-Saunders distribution. Duangsaphon (2014) [14] studied the Crack distribution in the regression-quantile estimation, Bayesian estimation. And also do the confidence interval estimation. Saengthong and Bodhisuwan (2014) [15] proposed the new two parameter Crack distribution by modifying the weighted parameter. Ngamkham (2019) [16] study about the three parameter Crack distribution and introduce a new algorithm to generate the random numbers by the composition method. Some relevant studies can be found in [17] and [18].

In this research, we propose a new four parameter family of distributions that generalizes the family three parameter Crack distribution and investigate the properties including to first four moments, parameter estimation by using the maximum likelihood estimators and method of moment estimation and evaluate the performance of the estimators by using bias.

The article is organized as follows. We first review the probability distribution function (pdf) of IG and LB and introduced you to know about Twice Length-Biased Inverse Gaussian $\left(\mathrm{LB}^{2}\right)$ distributions. Theoretical results about Generalization Crack distribution (GCR) are given in section 3. After that, Numerical results are shown in section 4. Finally, conclusions and discussion are reported in section 5 .

\section{Materials and Methods}

\subsection{Inverse Gaussian Distribution}

The probability distributions with support of $X$ on $(0, \infty)$. A random variable $X$ has the Inverse Gaussian distribution, if the probability density function is 


$$
f_{I G}(x ; \mu, \beta)=\sqrt{\frac{\beta}{2 \pi}} x^{-\frac{3}{2}} \exp \left\{-\frac{\beta(x-\mu)^{2}}{2 \mu^{2} x}\right\} ; x>0,
$$

where parameter $\mu>0$ is the mean and $\beta>0$ is the scale parameter of the distribution. We say that the probability density function above is the classical parametrization of the Inverse Gaussian distribution.

For the new parametrization of the Inverse Gaussian distribution, which is a two-parameter $\lambda>0, \theta>0$ family of continuous probability distributions with density function as follows

$$
f_{I G}(x ; \lambda, \theta)=\lambda \sqrt{\frac{\theta}{2 \pi}} x^{-\frac{3}{2}} \exp \left\{-\frac{(x-\lambda \theta)^{2}}{2 \theta x}\right\} ; x>0 .
$$

The relationship between classical parameters $\alpha, \beta$ and new parameters $\lambda, \theta$ can be written as follows

$$
\lambda=\frac{\beta}{\mu} \quad \text { and } \theta=\frac{\mu^{2}}{\beta} ; \mu=\lambda \theta \text { and } \beta=\lambda^{2} \theta .
$$

Let random variable $Y$ have $I G(\lambda, \theta)$ distribution. Then we get

$$
E[Y]=\lambda \theta \text { and } E\left[Y^{2}\right]=\lambda \theta^{2}(\lambda+1) .
$$

\subsection{Length-Biased Inverse Gaussian Distribution}

Let $X$ be a non-negative random variable having the continuous probability density function $f(\cdot)$ with a finite first moment $E[X]$. We say that $Y$ with probability density function $h(\cdot)$ has the length biased distribution associated with $X$, if its probability density function is given by the formula

$$
h(x)=\frac{x f(x)}{E[X]}, x>0
$$

From (1) and (2), then we get the Length Biased Inverse Gaussian distribution in term of new parametrization is given by the following formula

$$
f_{L B}(x ; \lambda, \theta)=\frac{1}{\theta \sqrt{2 \pi}} \sqrt{\frac{\theta}{x}} \exp \left\{-\frac{1}{2}\left(\sqrt{\frac{x}{\theta}}-\lambda \sqrt{\frac{\theta}{x}}\right)^{2}\right\} ; x>0 .
$$

We denote this distribution as $L B(\lambda, \theta)$ with mean $\theta(\lambda+1)$.

\subsection{Crack Distribution}

The Crack distribution is constructed by adding the weight parameter $p$ and including the two parameter of Inverse Gaussian distribution and two parameter of Length Biased Inverse Gaussian distribution. The formula is show as follows

$$
f_{C R}(x ; \lambda, \theta, p)=p f_{I G}(x ; \lambda, \theta)+(1-p) f_{L B}(x ; \lambda, \theta)
$$

where $\lambda>0, \theta>0$ and $0 \leq p \leq 1$. The probability density function of three-parameter Crack distribution is given by the following formula

$$
f_{C R}(x ; \lambda, \theta, p)=\frac{1}{\theta \sqrt{2 \pi}}\left[p \lambda\left(\frac{\theta}{x}\right)^{\frac{3}{2}}+(1-p)\left(\frac{\theta}{x}\right)^{\frac{1}{2}}\right] \exp \left\{-\frac{1}{2}\left(\sqrt{\frac{x}{\theta}}-\lambda \sqrt{\frac{\theta}{x}}\right)^{2}\right\} ; x>0,
$$

where $\lambda>0, \theta>0$ and $0 \leq p \leq 1$. We denote this distribution as $C R(\lambda, \theta, p)$.

The cumulative distribution function of three-parameter Crack distribution is

$$
F_{C R}(\lambda, \theta, p)=\Phi\left(\sqrt{\frac{x}{\theta}}-\lambda \sqrt{\frac{\theta}{x}}\right)-1-2 p e^{2 \lambda}\left[1-\Phi\left(\sqrt{\frac{x}{\theta}}-\lambda \sqrt{\frac{\theta}{x}}\right)\right] ; x>0,
$$

where $\Phi(x)$ is the standard normal distribution function.

The connection of the probability density functions of Inverse Gaussian distribution, Length Biased Inverse Gaussian distribution, Birnbaum-Saunders distribution with the Crack distribution is

$$
f_{C R}(x ; \lambda, \theta, p)= \begin{cases}f_{I G}(x ; \lambda, \theta), & p=1, \\ f_{B S}(x ; \lambda, \theta), & p=\frac{1}{2}, \\ f_{L B}(x ; \lambda, \theta), & p=0,\end{cases}
$$

where $\lambda>0, \theta>0$ and $0 \leq p \leq 1$.

\subsection{Twice Length-Biased Inverse Gaussian Distribution}

Let random variable $X$ have $L B(\lambda, \theta)$ distribution and $T$ have $I G(\lambda, \theta)$ distribution. According to formula (2), a nonnegative random variable $Y$ with density function $h(\cdot)$ has the length biased distribution associated with $X$, if its density function is given by the formula

$$
f_{L B^{2}}(x)=\frac{x f_{L B}(x)}{E(X)}=\frac{x \frac{x f_{I G}(x)}{E(T)}}{E(X)}=\frac{x^{2} f_{I G}(x)}{E(T) E(X)}
$$

The Twice Length Biased Inverse Gaussian distribution, which we consider in this article, is a two-parameter $\lambda>0, \theta>0$ family of continuous probability distributions with density function as

$$
\begin{aligned}
f_{L B^{2}}(x) & =\frac{x^{2} f_{I G}(x)}{E(T) E(X)} \\
& =\frac{x^{2}\left(\lambda \sqrt{\frac{\theta}{2 \pi}} x^{-\frac{3}{2}} \exp \left\{-\frac{1}{2}\left(\sqrt{\frac{x}{\theta}}-\lambda \sqrt{\frac{\theta}{x}}\right)^{2}\right\}\right)}{\lambda \theta^{2}(\lambda+1)} \\
& =\frac{\lambda}{\lambda \theta^{2}(\lambda+1)} \sqrt{\frac{\theta}{2 \pi}} x^{\frac{1}{2}} \exp \left\{-\frac{1}{2}\left(\sqrt{\frac{x}{\theta}}-\lambda \sqrt{\frac{\theta}{x}}\right)^{2}\right\} \\
& =\frac{1}{\theta \sqrt{2 \pi}(\lambda+1)} \sqrt{\frac{x}{\theta}} \exp \left\{-\frac{1}{2}\left(\sqrt{\frac{x}{\theta}}-\lambda \sqrt{\frac{\theta}{x}}\right)^{2}\right\}
\end{aligned}
$$

We denote this distribution as $L B^{2}(\lambda, \theta)$. 


\section{Generalization Crack Distribution}

\subsection{The Probability Distribution Function}

The Generalized CR lifetime distribution depends on four parameters. This distribution contains as special cases the four well-known aforementioned distributions, namely, the Crack distribution, the Birnbaum-Saunders distribution, the Inverse Gaussian distribution, and the Length Biased Inverse Gaussian distribution.

The Generalized Crack distribution is formed by adding one more weight parameter $q$ in the formila of Crack distribution and including the two parameter of Inverse Gaussian distribution, two parameter of Length Biased Inverse Gaussian distribution and two parameter of Twice Length Biased Inverse Gaussian distribution as follows

$f_{G C R}(x ; \lambda, \theta, p, q)=p f_{I G}(x ; \lambda, \theta)+q f_{L B}(x ; \lambda, \theta)+r f_{L B^{2}}(x ; \lambda, \theta)$,

Where $\lambda>0, \theta>0,0 \leq p \leq 1,0 \leq q \leq 1$ and $p+q+r=1$.

The probability density function of Generalized Crack distribution is given by

$$
\begin{aligned}
f_{G C R}(x ; \lambda, \theta, p, q) & =p f_{I G}(x ; \lambda, \theta)+q f_{L B}(x ; \lambda, \theta)+r f_{L B^{2}}(x ; \lambda, \theta) \\
& =p \lambda \sqrt{\frac{\theta}{2 \pi}} x^{-\frac{3}{2}} \exp \left\{-\frac{1}{2}\left(\sqrt{\frac{x}{\theta}}-\lambda \sqrt{\frac{\theta}{x}}\right)^{2}\right\} \\
& +\frac{q}{\theta \sqrt{2 \pi}} \sqrt{\frac{\theta}{x}} \exp \left\{-\frac{1}{2}\left(\sqrt{\frac{x}{\theta}}-\lambda \sqrt{\frac{\theta}{x}}\right)^{2}\right\} \\
& +\frac{r}{\theta \sqrt{2 \pi}(\lambda+1)} \sqrt{\frac{x}{\theta}} \exp \left\{-\frac{1}{2}\left(\sqrt{\frac{x}{\theta}}-\lambda \sqrt{\frac{\theta}{x}}\right)^{2}\right\} \\
& =\sqrt{\frac{\theta}{2 \pi}}\left[p \lambda x^{-\frac{3}{2}}+\frac{q}{\theta} x^{-\frac{1}{2}}+\frac{r}{(\lambda+1) \theta^{2}} x^{\frac{1}{2}}\right] \exp \left\{-\frac{1}{2}\left(\sqrt{\frac{x}{\theta}}-\lambda \sqrt{\frac{\theta}{x}}\right)^{2}\right\}
\end{aligned}
$$

where $\lambda>0, \theta>0,0 \leq p \leq 1,0 \leq q \leq 1$ and $p+q+r=1$. We denote this distribution as $\operatorname{GCR}(\lambda, \theta, p, q)$.

The relevance of the probability density functions of Crack distribution, Inverse Gaussian distribution, BirnbaumSaunders distribution, Length Biased Inverse Gaussian distribution, Twice Length Biased Inverse Gaussian distribution with the Generalized Crack distribution is

$$
f_{G C R}(x ; \lambda, \theta, p, q)=\left\{\begin{array}{cc}
f_{C R}(x ; \lambda, \theta), & q=1-p, r=0 \\
f_{I G}(x ; \lambda, \theta), & p=1, q=0, r=0 \\
f_{B S}(x ; \lambda, \theta), & p=\frac{1}{2}, q=\frac{1}{2}, r=0 \\
f_{L B}(x ; \lambda, \theta), & p=0, q=1, r=0 \\
f_{L B^{2}}(x ; \lambda, \theta), & p=0, q=0, r=1
\end{array}\right.
$$

where $\lambda>0, \theta>0,0 \leq p \leq 1,0 \leq q \leq 1$ and $p+q+r=1$.

Note that the connection between Generalized Crack distribution, Inverse Gaussian distribution, Length Biased Inverse Gaussian distribution and Twice Length Biased Inverse Gaussian distribution distributions can be illustrate in alternative way.

Let $X_{1}, X_{2}$ and $X_{3}$ are independent random variables such that $X_{1}$ has $\operatorname{IG}(\lambda, \theta)$ distribution, $X_{2}$ has $L B(\lambda, \theta)$ distribution and $X_{3}$ has $L B^{2}(\lambda, \theta)$ distribution, $0 \leq p \leq 1,0 \leq q \leq 1$ and $p+q+r=1$.

Consider the new random variable $X$ such that

$$
X= \begin{cases}X_{1} & \text { with probability } p, \\ X_{2} & \text { with probability } q, \\ X_{3} & \text { with probability } r,\end{cases}
$$

then $X$ follows the $\operatorname{GCR}(\lambda, \theta, p)$ distribution. This is the reason why we say that $X$ is a mixture of $X_{1}, X_{2}$ and $X_{3}$.

\subsection{The First Four Moments}

The moment generating function of $X \sim \operatorname{GCR}(\lambda, \theta, p, q)$ is

$\phi_{G C R}(t)=e^{\lambda(1-\sqrt{1-2 \theta t})}\left[p+\frac{q}{\sqrt{1-2 \theta t}}+\frac{r}{(\lambda+1)(1-2 \theta t)}\left(\lambda+\frac{1}{2 \sqrt{1-2 \theta t}}\right)\right]$

Proof Let $X$ be a $G C R(\lambda, \theta, p, q)$ distributed random variable; then

$$
\begin{aligned}
\phi_{G C R}(t) & =E\left\lfloor e^{t x}\right\rfloor \\
& =\int_{0}^{\infty} e^{t x} f_{G C R}(x, \lambda, \theta, p, q) d x \\
& =\int_{0}^{\infty} e^{t x} \sqrt{\frac{\theta}{2 \pi}}\left[p \lambda x^{-\frac{3}{2}}+\frac{q}{\theta} x^{-\frac{1}{2}}+\frac{r}{(\lambda+1) \theta^{2}} x^{\frac{1}{2}}\right] \exp \left\{-\frac{1}{2}\left(\sqrt{\frac{x}{\theta}}-\lambda \sqrt{\frac{\theta}{x}}\right)^{2}\right\} d x \\
& =p \lambda e^{\lambda} \sqrt{\frac{\theta}{2 \pi}} \int_{0}^{\infty} x^{-\frac{3}{2}} \exp \left\{-\left(\frac{1}{2 \theta}-t\right) x-\frac{\left(\lambda^{2} \theta / 2\right)}{x}\right\} d x \\
& +\frac{q e^{\lambda}}{\theta} \sqrt{\frac{\theta}{2 \pi}} \int_{0}^{\infty} x^{-\frac{1}{2}} \exp \left\{-\left(\frac{1}{2 \theta}-t\right) x-\frac{\left(\lambda^{2} \theta / 2\right)}{x}\right\} d x \\
& +\frac{r e^{\lambda}}{(\lambda+1) \theta^{2}} \sqrt{\frac{\theta}{2 \pi}} \int_{0}^{\infty} x^{\frac{1}{2}} \exp \left\{-\left(\frac{1}{2 \theta}-t\right) x-\frac{\left(\lambda^{2} \theta / 2\right)}{x}\right\} d x
\end{aligned}
$$

Therefore

$$
\begin{aligned}
\phi_{G C R}(t) & =p e^{\lambda} \exp \{-\lambda \sqrt{1-2 \theta t}\}+\frac{q e^{\lambda}}{\sqrt{1-2 \theta t}} \exp \{-\lambda \sqrt{1-2 \theta t}\} \\
& +\frac{r e^{\lambda}}{(\lambda+1)(1-2 \theta t)}\left[\lambda+\frac{1}{2 \sqrt{1-2 \theta t}}\right] \exp \{-\lambda \sqrt{1-2 \theta t}\} \\
& =e^{\lambda(1-\sqrt{1-2 \theta t})}\left[p+\frac{q}{\sqrt{1-2 \theta t}}+\frac{r}{(\lambda+1)(1-2 \theta t)}\left(\lambda+\frac{1}{2 \sqrt{1-2 \theta t}}\right)\right]
\end{aligned}
$$

Where $t<1 / 2 \theta$

Hence, the first four moments of $X$ is given by

$$
\begin{aligned}
E(X) & =q \theta+\frac{r \theta}{2(\lambda+1)}+\frac{2 r \theta\left(\frac{1}{2}+\lambda\right)}{\lambda+1}+\lambda \theta\left(p+q+\frac{r\left(\frac{1}{2}+\lambda\right)}{\lambda+1}\right) \\
E\left(X^{2}\right) & =3 q \theta^{2}+\frac{7 r \theta^{2}}{2(\lambda+1)}+\frac{8 r \theta^{2}\left(\frac{1}{2}+\lambda\right)}{\lambda+1}+\lambda \theta^{2}\left(p+q+\frac{r\left(\frac{1}{2}+\lambda\right)}{\lambda+1}\right) \\
& +\lambda^{2} \theta^{2}\left(p+q+\frac{r\left(\frac{1}{2}+\lambda\right)}{\lambda+1}\right)+2 \lambda \theta\left(q \theta+\frac{r \theta}{2(\lambda+1)}+\frac{2 r \theta\left(\frac{1}{2}+\lambda\right)}{\lambda+1}\right)
\end{aligned}
$$




$$
\begin{aligned}
& E\left(X^{3}\right)=15 q \theta^{3}+\frac{57 r \theta^{3}}{2(\lambda+1)}+\frac{48 r \theta^{3}\left(\frac{1}{2}+\lambda\right)}{\lambda+1}+3 \lambda \theta^{3}\left(p+q+\frac{r\left(\frac{1}{2}+\lambda\right)}{\lambda+1}\right) \\
& +3 \lambda^{2} \theta^{3}\left(p+q+\frac{r\left(\frac{1}{2}+\lambda\right)}{\lambda+1}\right)+\lambda^{3} \theta^{3}\left(p+q+\frac{r\left(\frac{1}{2}+\lambda\right)}{\lambda+1}\right) \\
& +3 \lambda \theta^{2}\left(q \theta+\frac{r \theta}{2(\lambda+1)}+\frac{2 r \theta\left(\frac{1}{2}+\lambda\right)}{\lambda+1}\right)+3 \lambda^{2} \theta^{2}\left(q \theta+\frac{r \theta}{2(\lambda+1)}+\frac{2 r \theta\left(\frac{1}{2}+\lambda\right)}{\lambda+1}\right) \\
& +3 \lambda \theta\left(3 q \theta^{2}+\frac{7 r \theta^{2}}{2(\lambda+1)}+\frac{8 r \theta^{2}\left(\frac{1}{2}+\lambda\right)}{\lambda+1}\right) \\
& E\left(X^{4}\right)=105 q \theta^{4}+\frac{561 r \theta^{4}}{2(\lambda+1)}+\frac{384 r \theta^{4}\left(\frac{1}{2}+\lambda\right)}{\lambda+1}+15 \lambda \theta^{4}\left(p+q+\frac{r\left(\frac{1}{2}+\lambda\right)}{\lambda+1}\right) \\
& +15 \lambda^{2} \theta^{4}\left(p+q+\frac{r\left(\frac{1}{2}+\lambda\right)}{\lambda+1}\right)+6 \lambda^{3} \theta^{4}\left(p+q+\frac{r\left(\frac{1}{2}+\lambda\right)}{\lambda+1}\right) \\
& +\lambda^{4} \theta^{4}\left(p+q+\frac{r\left(\frac{1}{2}+\lambda\right)}{\lambda+1}\right)+12 \lambda \theta^{3}\left(q \theta+\frac{r \theta}{2(\lambda+1)}+\frac{2 r \theta\left(\frac{1}{2}+\lambda\right)}{\lambda+1}\right) \\
& +12 \lambda^{2} \theta^{3}\left(q \theta+\frac{r \theta}{2(\lambda+1)}+\frac{2 r \theta\left(\frac{1}{2}+\lambda\right)}{\lambda+1}\right)+4 \lambda^{3} \theta^{3}\left(q \theta+\frac{r \theta}{2(\lambda+1)}+\frac{2 r \theta\left(\frac{1}{2}+\lambda\right)}{\lambda+1}\right)
\end{aligned}
$$

Let $X \square G C R(x ; \lambda, \theta, p, q)$ with mean $\mu=E(X)$ and variance $\sigma^{2}=V(X)$. Therefore,

And

$$
\mu=E(X)=p \lambda \theta+q \theta(\lambda+1)+\frac{r \theta(2 \lambda+3)}{2}
$$

$$
\begin{aligned}
\sigma^{2}= & V(X) \\
& =E\left(X^{2}\right)-[E(x)]^{2} \\
& =p \lambda \theta^{2}(\lambda+1)+q \theta^{2}[\lambda(\lambda+2)+(\lambda+3)] \\
& +\frac{r \theta^{2}\left[2 \lambda^{2}(\lambda+3)+5 \lambda(\lambda+4)+3(\lambda+5)\right]}{2(\lambda+1)} \\
& -\left[p \lambda \theta+q \theta(\lambda+1)+\frac{r \theta(2 \lambda+3)}{2}\right]^{2} \\
& =p \lambda \theta^{2}[1+\lambda(1-p)-2 q(\lambda+1)] \\
& +q \theta^{2}\left[\left(\lambda^{2}+3 \lambda+3\right)-r(\theta+1)(2 \theta+3)-q(\lambda+1)^{2}\right] \\
& +\frac{r \theta^{2}\left[r\left(-3 \lambda-4 \lambda^{3}+9\right)-2 \lambda(2 p(2 \lambda-3)(\lambda+1)-\lambda(2 \lambda-8 r+11)-23)+30\right]}{4(\lambda+1)}
\end{aligned}
$$

\subsection{The Characteristic Function}

The characteristic function of $X \sim \operatorname{GCR}(\lambda, \theta, p, q)$ is

$\varphi_{G C R}(i t)=e^{\lambda(1-\sqrt{1-2 \theta i t})}\left[p+\frac{q}{\sqrt{1-2 \theta i t}}+\frac{r}{(\lambda+1)(1-2 \theta i t)}\left(\lambda+\frac{1}{2 \sqrt{1-2 \theta i t}}\right)\right]$
Proof Let $X$ be a $G C R(\lambda, \theta, p, q)$ distributed random variable; then

$$
\begin{aligned}
& \varphi_{G C R}(i t)=E\left[e^{i t X}\right] \\
& =\int_{0}^{\infty} e^{i t X} f_{G C R}(x, \lambda, \theta, p, q) d x \\
& =\int_{0}^{\infty} e^{i t X} \sqrt{\frac{\theta}{2 \pi}}\left[p \lambda x^{-\frac{3}{2}}+\frac{q}{\theta} x^{-\frac{1}{2}}+\frac{r}{(\lambda+1) \theta^{2}} x^{\frac{1}{2}}\right] \exp \left\{-\frac{1}{2}\left(\sqrt{\frac{x}{\theta}}-\lambda \sqrt{\frac{\theta}{x}}\right)^{2}\right\} d x \\
& =p \lambda e^{\lambda} \sqrt{\frac{\theta}{2 \pi}} \int_{0}^{\infty} x^{-\frac{3}{2}} \exp \left\{-\left(\frac{1}{2 \theta}-i t\right) x-\frac{\left(\lambda^{2} \theta / 2\right)}{x}\right\} d x \\
& +\frac{q e^{\lambda}}{\theta} \sqrt{\frac{\theta}{2 \pi}} \int_{0}^{\infty} x^{-\frac{1}{2}} \exp \left\{-\left(\frac{1}{2 \theta}-i t\right) x-\frac{\left(\lambda^{2} \theta / 2\right)}{x}\right\} d x \\
& +\frac{r e^{\lambda}}{(\lambda+1) \theta^{2}} \sqrt{\frac{\theta}{2 \pi}} \int_{0}^{\infty} x^{\frac{1}{2}} \exp \left\{-\left(\frac{1}{2 \theta}-i t\right) x-\frac{\left(\lambda^{2} \theta / 2\right)}{x}\right\} d x \\
& =p e^{\lambda} \exp \{-\lambda \sqrt{1-2 \theta i t}\}+\frac{q e^{\lambda}}{\sqrt{1-2 \theta i t}} \exp \{-\lambda \sqrt{1-2 \theta i t}\} \\
& +\left[\frac{r \lambda e^{\lambda}}{(\lambda+1)(1-2 \theta i t)}+\frac{r e^{\lambda}}{2(\lambda+1)(1-2 \theta i t) \sqrt{1-2 \theta i t}}\right] \exp \{-\lambda \sqrt{1-2 \theta i t}\} \\
& =e^{\lambda(1-\sqrt{1-2 \theta i t})}\left[p+\frac{q}{\sqrt{1-2 \theta i t}}+\frac{r}{(\lambda+1)(1-2 \theta i t)}\left(\lambda+\frac{1}{2 \sqrt{1-2 \theta i t}}\right)\right]
\end{aligned}
$$

Where $t \in \square$

\subsection{The Method of Moment Estimation}

Let $X_{1}, X_{2}, \ldots, X_{n}$ be a sample from $\operatorname{GCR}(\lambda, \theta, p, q)$ distribution and $x_{1}, x_{2}, \ldots, x_{n}$ be the sample values. Denote the sample noncentral moments as

$$
\begin{aligned}
& m_{1}=\frac{1}{n} \sum_{i=1}^{n} x_{i} \\
& m_{2}=\frac{1}{n} \sum_{i=1}^{n} x_{i}^{2} \\
& m_{3}=\frac{1}{n} \sum_{i=1}^{n} x_{i}^{3} \\
& m_{4}=\frac{1}{n} \sum_{i=1}^{n} x_{i}^{4} .
\end{aligned}
$$

Then the equations for the Method of Moments are:

$$
\begin{aligned}
& E(X)=m_{1} \\
& E\left(X^{2}\right)=m_{2} \\
& E\left(X^{3}\right)=m_{3} \\
& E\left(X^{4}\right)=m_{4},
\end{aligned}
$$

where $E(X), E\left(X^{2}\right), E\left(X^{3}\right)$, and $E\left(X^{4}\right)$ as functions of the parameters $\lambda, \theta, p$, and $q$ in section III. $B$. Unfortunately, the Method of Moments equations cannot be solved in closed form so, we used MatLab to solve the system of nonlinear algebraic equations numerically. We using function lsqnonlin to solved the the system of nonlinear algebraic equations numerically of the equations for the Method of Moments.

\subsection{The Maximum Likelihood Estimation}

Let $f(x \mid \theta)$ be a probability density function (p.d.f.) where $\theta$ is a vector of parameters. Let $X_{1}, X_{2}, \ldots, X_{n} \sim f(x \mid \theta)$. The likelihood function can be written as:

$$
L\left(\theta \mid x_{1}, x_{2}, \ldots, x_{n}\right)=\prod_{i=1}^{n} f\left(x_{i} \mid \theta\right)
$$


The maximum Likelihood Estimator (M.L.E.), $\hat{\theta}$ is the value of $\theta$ that maximizes $L\left(\theta \mid x_{1}, x_{2}, \ldots, x_{n}\right)$

Let $X \sim G C R(\lambda, \theta, p, q)$, the likelihood equations are:

$$
\begin{gathered}
\frac{\partial l}{\partial \lambda}=n-\sum_{i=1}^{n}\left(\frac{p \theta^{2}-\frac{r x_{i}^{2}}{(\lambda+1)^{2}}}{p \lambda \theta^{2}+q \theta x_{i}+\frac{r}{(\lambda+1)} x_{i}^{2}}\right)-\lambda \theta \sum_{i=1}^{n} \frac{1}{x_{i}}=0 \\
\frac{\partial l}{\partial \theta}=\frac{n}{2 \theta}+\frac{2 n}{\theta}-\sum_{i=1}^{n}\left(\frac{2 p \lambda \theta+q x_{i}}{p \lambda \theta^{2}+q \theta x_{i}+\frac{r}{(\lambda+1)} x_{i}^{2}}\right)+\frac{1}{2 \theta^{2}} \sum_{i=1}^{n} x_{i}-\frac{\lambda^{2}}{2} \sum_{i=1}^{n} \frac{1}{x_{i}} \\
\frac{\partial l}{\partial p}=-\sum_{i=1}^{n}\left(\frac{\lambda \theta^{2}}{p \lambda \theta^{2}+q \theta x_{i}+\frac{r}{(\lambda+1)} x_{i}^{2}}\right) \\
\frac{\partial l}{\partial q}=-\sum_{i=1}^{n}\left(\frac{\theta x_{i}}{p \lambda \theta^{2}+q \theta x_{i}+\frac{r}{(\lambda+1)} x_{i}^{2}}\right)
\end{gathered}
$$

\section{Numerical Results}

\subsection{Simulation Study}

A simulation study is performed to evaluate the performance of MME and MLE estimator for the Generalized Crack Random Number that generate by the Composition method. To compare two methods of point estimation including with MME and MLE, we consider the bias of estimators. The simulations were carried out in R statistical software and MATLAB. For each point estimation method, we use all combinations of $n=100,500,1000,5000$. And the combination of parameter are $l, q=1,2$ and $p, q=1 / 3$. The results were report to investigated the behavior of estimators by using bias. We report the results of the estimated parameter for both MME

\begin{tabular}{|c|c|c|c|c|c|c|c|c|c|c|}
\hline \multirow{2}{*}{$\lambda$} & \multirow{2}{*}{$\theta$} & \multirow[b]{2}{*}{$\mathrm{n}$} & \multicolumn{4}{|c|}{ MME } & \multicolumn{4}{|c|}{ MLE } \\
\hline & & & $\hat{\lambda}$ & $\hat{\theta}$ & $\hat{q}$ & $\widehat{q}$ & $\hat{\lambda}$ & $\hat{\theta}$ & $\hat{q}$ & $\bar{q}$ \\
\hline \multirow[t]{4}{*}{1} & \multirow[t]{4}{*}{1} & 100 & 2.2454 & 0.6881 & \begin{tabular}{|c|c|c|}
0.1165 \\
\end{tabular} & 0.5296 & 1.9999 & 0.7630 & \begin{tabular}{|c|}
0.1178 \\
\end{tabular} & 0.3061 \\
\hline & & 500 & 3.5394 & 0.4437 & 0.1155 & 0.5227 & 1.9553 & 0.7650 & 0.1169 & 0.3025 \\
\hline & & 1000 & 2.2109 & 0.6921 & 0.1161 & 0.5370 & 1.9765 & 0.7695 & 0.1174 & 0.2994 \\
\hline & & 5000 & 2.2144 & 0.6901 & 0.1161 & 0.5366 & 1.9883 & 0.7687 & 0.1175 & 0.2991 \\
\hline \multirow[t]{4}{*}{1} & \multirow[t]{4}{*}{2} & 100 & 2.2282 & 0.6996 & 0.1211 & 0.5721 & 2.6464 & 0.8466 & 0.1237 & 0.2899 \\
\hline & & 500 & 2.1871 & 0.6918 & 0.1200 & 0.5723 & 2.5650 & 0.8515 & 0.1225 & 0.2830 \\
\hline & & 1000 & 2.1841 & 0.7028 & 0.1206 & 0.5897 & 2.6326 & 0.8521 & \begin{tabular}{|c|c|c|} 
\\
\end{tabular} & 0.2796 \\
\hline & & 5000 & 2.1934 & 0.6987 & 0.1206 & 0.5836 & 2.6329 & 0.8517 & \begin{tabular}{|c|c|c|} 
\\
\end{tabular} & 0.2814 \\
\hline \multirow[t]{4}{*}{2} & \multirow[t]{4}{*}{1} & 100 & 2.1841 & 0.7201 & 0.1264 & 0.6271 & 3.0997 & 0.8258 & $\begin{array}{c}0.1309 \\
\end{array}$ & 0.2717 \\
\hline & & 500 & 2.1354 & 0.7102 & \begin{tabular}{|c|c|c|} 
\\
\end{tabular} & 0.6305 & 2.9717 & 0.8159 & $\begin{array}{c}0.1292 \\
\end{array}$ & 0.2636 \\
\hline & & 1000 & 2.1353 & 0.7219 & $\begin{array}{c}0.1259 \\
\end{array}$ & 0.6510 & 2.9817 & 0.8148 & 0.1303 & 0.2603 \\
\hline & & 5000 & 2.1475 & 0.7157 & 0.1258 & 0.6398 & 2.9584 & 0.8118 & 0.1302 & 0.2631 \\
\hline \multirow[t]{4}{*}{2} & \multirow[t]{4}{*}{2} & 100 & 3.3571 & 0.5317 & 0.1327 & 0.6915 & 4.5763 & 0.8956 & 0.1400 & 0.2539 \\
\hline & & 500 & 2.0688 & 0.7373 & 0.1312 & 0.6948 & 4.2552 & 0.8870 & 0.1378 & 0.2455 \\
\hline & & 1000 & 3.0495 & 0.5761 & 0.1320 & 0.7173 & 4.2782 & 0.8864 & 0.1391 & 0.2429 \\
\hline & & 5000 & 2.0855 & 0.7408 & \begin{tabular}{|c|}
0.1319 \\
\end{tabular} & 0.7023 & 4.2528 & 0.8846 & 0.1390 & 0.2458 \\
\hline
\end{tabular}
and MLE in Table I and report the eatimated bias of four parameters in Table II as follows

Table I. The MME and MLE estimators of $l, q, p$ and $q$

From Table I shows the MME and MLE estimators $l, q, p$ and $q$, we can see that the performance of estimators of parameter $\lambda, \theta, p$ and $q$ are good for large sample sizes

\begin{tabular}{|c|c|c|c|c|c|c|c|c|c|c|}
\hline \multirow{3}{*}{$\lambda$} & \multirow{3}{*}{$\theta$} & \multirow{3}{*}{$\mathrm{n}$} & \multicolumn{8}{|c|}{ Bias } \\
\hline & & & \multicolumn{4}{|c|}{ MNE } & \multicolumn{4}{|c|}{ MLE } \\
\hline & & & $\lambda-\hat{\lambda}$ & $\theta-\hat{\theta}$ & $p-\hat{p}$ & $\mathrm{q}-\widehat{q}$ & $\lambda-\hat{\lambda}$ & $\theta-\hat{\theta}$ & $p-\hat{p}$ & $q-\hat{q}$ \\
\hline \multirow[t]{4}{*}{1} & \multirow[t]{4}{*}{1} & 100 & 1.2454 & -0.3119 & -0.2169 & 0.1963 & \begin{tabular}{|c|}
0.9999 \\
\end{tabular} & -0.2370 & -0.2155 & -0.0273 \\
\hline & & 500 & 2.5394 & -0.5563 & -0.2178 & 0.1893 & 0.9553 & -0.2350 & -0.2164 & -0.0309 \\
\hline & & 1000 & 1.2109 & -0.3079 & -0.2173 & 0.2036 & 0.9765 & -0.2305 & -0.2159 & -0.0339 \\
\hline & & 5000 & 1.2144 & -0.3099 & -0.2172 & 0.2033 & 0.9883 & -0.2313 & -0.2159 & -0.0342 \\
\hline \multirow[t]{4}{*}{1} & \multirow[t]{4}{*}{2} & 100 & 1.2282 & -1.3004 & -0.2122 & 0.2388 & 1.6464 & -1.1534 & -0.2096 & -0.0434 \\
\hline & & 500 & 1.1871 & -1.3082 & -0.2134 & 0.2390 & 1.5650 & -1.1485 & -0.2108 & -0.0503 \\
\hline & & 1000 & 1.1841 & -1.2972 & -0.2127 & 0.2564 & 1.6326 & -1.1479 & -0.2101 & -0.0537 \\
\hline & & 5000 & 1.1934 & -1.3013 & -0.2127 & \begin{tabular}{|c|c|}
0.2503 \\
\end{tabular} & 1.6329 & -1.1483 & -0.2101 & -0.0520 \\
\hline \multirow[t]{4}{*}{2} & \multirow[t]{4}{*}{1} & 100 & 0.1841 & -0.2799 & -0.2069 & 0.2937 & \begin{tabular}{|l|l|}
1.0997 \\
\end{tabular} & -0.1742 & -0.2024 & -0.0616 \\
\hline & & 500 & 0.1354 & -0.2898 & -0.2082 & 0.2972 & 0.9717 & -0.1841 & -0.2041 & -0.0698 \\
\hline & & 1000 & 0.1353 & -0.2781 & -0.2074 & 0.3176 & 0.9817 & -0.1852 & -0.2031 & -0.0730 \\
\hline & & 5000 & 0.1475 & -0.2843 & -0.2075 & 0.3064 & 0.9584 & -0.1882 & -0.2031 & -0.0702 \\
\hline \multirow[t]{4}{*}{2} & \multirow[t]{4}{*}{2} & 100 & 1.3571 & -1.4683 & -0.2007 & 0.3581 & 2.5763 & -1.1044 & -0.1934 & -0.0795 \\
\hline & & 500 & 0.0688 & -1.2627 & -0.2021 & 0.3614 & 2.2552 & -1.1130 & -0.1955 & -0.0878 \\
\hline & & 1000 & 1.0495 & -1.4239 & -0.2013 & 0.3840 & 2.2772 & -1.1136 & -0.1942 & -0.0904 \\
\hline & & 5000 & 0.0855 & -1.2592 & -0.2214 & 0.3690 & 2.2528 & -1.1154 & -0.1944 & -0.0875 \\
\hline
\end{tabular}

which are 1000 and 5000 but the small sample sizes which are 100 and 500 are poor for both method MME and MLE.

Table II. The bias of $l, q, p$ and $q$

Table II shows the The bias of $l, q, p$ and $q$, we can see that the method of moment estimators has generally overestimate; almost every estimated value provided the negative value except $\lambda$ and $\mathrm{q}$ for all combinations. While the MLE provided the different results, the parameters $\lambda$ still overestimate but parameter q was underestimate.

When the sample sizes increase, the simulated bias corresponds to the theoretical background as it is a decreasing function of sample sizes $\mathrm{n}$. That is, when sample sizes increase, the amount of the bias decreases and tends to zero. From Table II when we increase sample sizes from 100 to 5000 in every combination, bias of each estimation is decreasing when sample sizes increase.

\section{Discussion}

This research contains new contributions as the following:

1. Motivating hopeful practitioners to protect the industrial or financial damages before the lifetime expired. It may also protect lives safe due to the fact that workers who do not know the lifetime of the equipment or items that need to be used in the work may cause direct and indirect damage. The lifetime distribution with the high performance of parameter estimate can prevent damage.

2. Improving statistical knowledge about lifetime distribution.

\section{Conclusion}

This new Crack Lifetime distribution is useful in many areas for example Engineering, Physics, Economics and Statistics. In this research, we propose a new four parameter family of distributions that generalizes the family three parameter Crack distribution called Generalized Crack distribution. In addition, we investigate the properties including to first four moments, parameter estimation by using the maximum likelihood estimators and method of moment estimation and evaluate the performance of the estimators by using bias. Since in this situation, the MLE estimator has the performance better than MME so, we recommended to use MLE estimator for the Generalized Crack distribution. 


\section{Acknowledgment}

The authors gratefully acknowledge the reviewers for suggestions which lead to considerable improvements in the manuscript and thank the Department of Mathematics and Statistics Faculty of Science and Technology Thammasat University for our financial support.

\section{References}

[1] E. Schrodinger, "Zur theorie der fall-und steigversuche an teilchenn mit brownscher bewegung," Physikalische Zeitschrift 16, 289-295 (1915).

[2] A. Wald, Sequential Analysis (New York, John Wiley and Sons, 1947)

[3] M. C. K. Tweedie, "Statistical properties of Inverse Gaussian distributions. I, II,” Ann. Math. Statist. 28 362-377, 696-705 (1957).

[4] J. Shuster, "On the Inverse Gaussian distribution function. " J. Amer. Statist. Assoc. 63, 1514-1516 (1968).

[5] R. S. Chhikara and J. L. Folks, The Inverse Gaussian Distribution: Theory, Methodology, and Applications (Marcel Dekker Inc., New York, 1989).

[6] Y. P. Chaubey, D. Sen, and K. K. Saha, " On testing the coefficient of variation in an inverse Gaussian population. "Statist. Probab. Lett. 90, 121-128 (2014).

[7] M. Ahsanullah and S. N. U. A. Kirmani, "A characterization of the Wald distribution,” Naval Res. Logist. Quart. 31 (1), 155-158 (1984).

[8] R. Khattree, " Characterization of Inverse-Gaussian and gamma distributions through their length-biased distribution," IEEE Reliabilitty 38, 610-611 (1989).

[9] G. P. Patil and C. R. Rao, Weighted distributions and a survey of their applications. In: Applications of Statistics, P. R. Krishnaiah, Eds., North-Holland Publishing Co., 383-405 (1977).

[10] B. Jørgensen, V. Seshadri, and G. A. Whitmore, " On the mixture of the Inverse Gaussian distribution with its complementary reciprocal," Scand. J. Statist. 18 (1), 77-89 (1991).

[11] R. C. Gupta and H. O. Akman, "On the reliability studies of a weighted Inverse Gaussian model,” J. Statist. Plann. Inference 48 (1), 69-83 (1995).

[12] R. C. Gupta and H. O. Akman, " Bayes estimation in a mixture Inverse Gaussian model," Ann. Inst. Statist. Math. 47 (3), 493-503 (1995).

[13] I. N. Volodin and O. A. Dzhungurova, On limit distributions emerging in the generalized Birnbaum-Saunders model. In: Proceedings of the 19th Seminar on Stability Problems for Stochastic Models, Part I (Vologda, 1998) 99, 1348-1366 (2000).

[14] M. Duangsaphon, Improved statistical inference for three-parameter Crack lifetime distribution. Ph. D. Thesis, Thammasat University (2014).

[15] P. Saengthong and W. Bodhisuwan, " A new two-parameter Crack distribution," Applied Sciences 14 (8), 758-766 (2014).

[16] Ngamkham. "On the Crack Random Numbers Generation Procedure," Lobachevskii Journal of Mathematics, (2019).

[17] Pulu Han, Nonlinear Mechanics Study of Concrete T-beam Bridge With Cracking Damage Based on Numerical Simulation, International Journal of Circuits, Systems and Signal Processing, Volume 14, 249254 (2020).

[18] Z. Kala, A. Omishore, S. Seitl, M. Krejsa, J. Kala, Identification of Variation Coefficient of Equivalent Stress Range of Steel Girders with Cracks, International Journal of Mechanics, Volume 13, $69-78$ (2019).

\section{Creative Commons Attribution License 4.0 (Attribution 4.0 International, CC BY 4.0)}

This article is published under the terms of the Creative Commons Attribution License 4.0 https://creativecommons.org/licenses/by/4.0/deed.en US 